\title{
Development and Analysis of a Sensitivity Matrix of a 3-phase Voltage Unbalance Factor
}

\author{
Young-Jin Kim, Member, IEEE
}

\begin{abstract}
Unbalanced phase voltages in electricity networks prevent efficient and stable delivery of power and energy services from utility companies to end-users, and vice versa. While variations in voltage magnitude and power loss have been studied extensively, little attention has been paid to the development of linearized expressions to account for the variation in voltage unbalance. Using a standard definition of the voltage unbalance factor (VUF), this study is the first, to the best knowledge of the authors, in which a VUF sensitivity matrix is mathematically developed and analyzed with respect to variations in 3-phase (3-ph) voltages and, consequently, 3-ph unbalanced load demand. To demonstrate its utility, we performed simulation case studies and compared the estimates made using the matrix to those from a power flow calculation algorithm. The VUF sensitivity matrix is expected to have a variety of practical applications for power systems, such as optimal scheduling and real-time control, which enables improvement in network power quality.
\end{abstract}

Index Terms-distribution network, power flow calculation, power quality, sensitivity matrix, voltage unbalance factor (VUF)

\section{INTRODUCTION}

$\mathrm{U}^{\mathrm{N}}$ NBALANCED voltages and, consequently, currents usually occur when single-phase $(1-p h)$ loads are connected between two phases, or between one phase and the neutral. In particular, 1-ph building loads are randomly connected to any two points of three-phase (3-ph) power lines. Therefore, it frequently occurs that one or two phases are heavily charged, causing large unbalances in phase voltages. It is difficult to change load connections, as this requires retrofitting of building electricity systems. Voltage unbalance also can occur in 3-ph loads that are operating normally when the line impedances are asymmetrical. Voltage unbalance is considered to be one of the main causes of power quality problems. It is also detrimental to the performance of power equipment [1]. Under unbalanced voltage conditions, power networks incur more power losses and heating effects, rendering them less stable [2]. Voltage unbalance also has adverse effects on equipment such as induction motors and power electronics converters. For example, if the voltages on the stator of an induction motor are unbalanced, the winding insulation may fail prematurely or the operational efficiency can be reduced [3]. Voltage unbalances in AC-DC converters can cause low-order frequency power ripples, leading to current harmonics and poor DC-link voltage regulation [4]. The International Electrotechnical Commission (IEC) recommends that voltage unbalance in power grids should be limited to $2 \%$ [1]. Consequently, grid operators or electric utility companies need to optimize the operation of distributed energy resources (DERs) and controllable loads while taking variations in phase

Manuscript received September 17, 2017. This research was supported in part by the National Research Foundation of Korea (NRF) grant funded by the Korea government (MSIP) (NRF-2016R1E1A1A01941191). Paper no.

Y. Kim is with the Department of Electrical Engineering, Pohang University of Science and Technology (POSTECH), Pohang, Gyungbuk, 37673, Korea. voltage unbalances into consideration, so as to achieve stable and reliable operations of electric power networks in practice.

For the practical application, it is important to estimate how power inputs and outputs of the devices will affect voltage unbalance in the entire network. To make these estimates, it is necessary to undertake a sensitivity analysis of the voltage unbalance. However, while voltage magnitudes and power losses have been widely studied, little attention has been paid to developing a linearized expression of voltage unbalance. Previously, the effects of voltage unbalance on optimal power flow were analyzed using nonlinear optimization algorithms with iterative gradient methods, which are often computationally expensive. These methods also require additional effort to ensure convexity and hence avoid getting stuck in local optima.

In this paper, we propose a sensitivity matrix of the 3-ph voltage unbalance, based on a standard definition of the voltage unbalance factor (VUF). To the best knowledge of the authors, this study is the first in which a mathematical tool is developed to estimate a VUF sensitivity matrix that can be directly incorporated into linear programming (LP) or mixed-integer LP (MILP) algorithms. These algorithms have been widely used to comprehensively model and determine the optimal operation of complicated power systems mainly because convergence to the global optimum is guaranteed within a reasonable time using off-the-shelf software. Therefore, this incorporation enables grid operators or electric utility companies to easily determine optimal power flow or optimal operations of 1-ph DERs and loads considering voltage unbalance variations. Case studies are performed to validate the estimates calculated using the sensitivity matrix by comparing them to those obtained from power flow calculations, where a backward-forward sweep (BFS) algorithm is used [5]. The comparison is valid as the BFS algorithm does not require a Jacobian or sensitivity matrix.

\section{SENSITIVITY MATRIX OF VUF}

The IEC defines the VUF as the ratio of negative sequence voltage $\left|V_{-}\right|$to positive sequence voltage $\left|V_{+}\right|$[6]. In (1), the 3-ph voltages at bus $k$ are expressed in rectangular coordinates. The derivation of the VUF at bus $k$ is shown in (2)-(5).

$$
\begin{aligned}
V_{a}^{k} & =E_{a}^{k}+j F_{a}^{k}, V_{b}^{k}=E_{b}^{k}+j F_{b}^{k}, \text { and } V_{c}^{k}=E_{c}^{k}+j F_{c}^{k} \\
V U F^{k} & =f\left(V_{a}^{k}, V_{b}^{k}, V_{c}^{k}\right)=g\left(E_{a}^{k}, F_{a}^{k}, E_{b}^{k}, F_{b}^{k}, E_{c}^{k}, F_{c}^{k}\right) \\
& =\left|\frac{V_{-}^{k}}{V_{+}^{k}}\right|=\left|\frac{V_{a}^{k}+\alpha^{2} V_{b}^{k}+\alpha V_{c}^{k}}{V_{a}^{k}+\alpha V_{b}^{k}+\alpha^{2} V_{c}^{k}}\right|=\left|\frac{n_{1}^{k}+j n_{2}^{k}}{d_{1}^{k}+j d_{2}^{k}}\right|=\left(\frac{n^{k}}{d^{k}}\right)^{1 / 2},
\end{aligned}
$$

where $\alpha=e^{j 2 \pi / 3}=\alpha_{r e}+j \alpha_{i m}$,

$\left[\begin{array}{l}n_{1}^{k} \\ n_{2}^{k} \\ d_{1}^{k} \\ d_{2}^{k}\end{array}\right]=\left[\begin{array}{cccccc}1 & \alpha_{r e} & \alpha_{r e} & 0 & \alpha_{i m} & -\alpha_{i m} \\ 0 & -\alpha_{i m} & \alpha_{i m} & 1 & \alpha_{r e} & \alpha_{r e} \\ 1 & \alpha_{r e} & \alpha_{r e} & 0 & -\alpha_{i m} & \alpha_{i m} \\ 0 & \alpha_{i m} & -\alpha_{i m} & 1 & \alpha_{r e} & \alpha_{r e}\end{array}\right] \cdot \mathbf{V}^{k}=\left[\begin{array}{c}\boldsymbol{\alpha}_{\mathbf{n 1}} \\ \boldsymbol{\alpha}_{\mathrm{n} 2} \\ \boldsymbol{\alpha}_{\mathrm{d} 1} \\ \boldsymbol{\alpha}_{\mathrm{d} 2}\end{array}\right] \cdot \mathbf{V}^{k}$,

and $\mathbf{V}^{k}=\left[\begin{array}{lll}\mathbf{E}^{k} & \mathbf{F}^{k}\end{array}\right]^{T}=\left[\begin{array}{llllll}E_{a}^{k} & E_{b}^{k} & E_{c}^{k} & F_{a}^{k} & F_{b}^{k} & F_{c}^{k}\end{array}\right]^{T}$. 
From (2), the variation in the VUF at bus $k$ can be estimated with respect to the change in $\mathbf{V}^{k}$ as

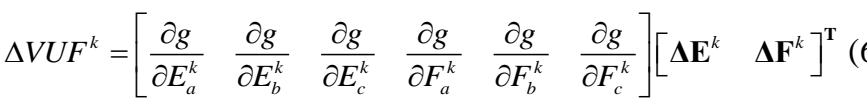

$$
\begin{aligned}
& =\mathbf{J}_{\mathbf{E F}}^{\mathbf{V U F}, k}\left[\begin{array}{cc}
\Delta \mathbf{E}^{k} & \Delta \mathbf{F}^{k}
\end{array}\right]^{\mathbf{T}}=\mathbf{J}_{\mathbf{E F}}^{\mathbf{V U F}, k} \boldsymbol{\Delta} \mathbf{V}^{k} .
\end{aligned}
$$

For $x=E_{a}{ }^{k}, E_{b}{ }^{k}, E_{c}{ }^{k}, F_{a}{ }^{k}, F_{b}{ }^{k}$, and $F_{c}{ }^{k}$, the partial derivatives in (6) can be represented as

$$
\frac{\partial g}{\partial x}=c^{k}\left\{\left(n_{1}^{k} \frac{\partial n_{1}^{k}}{\partial x}+n_{2}^{k} \frac{\partial n_{2}^{k}}{\partial x}\right) d^{k}-\left(d_{1}^{k} \frac{\partial d_{1}^{k}}{\partial x}+d_{2}^{k} \frac{\partial d_{2}^{k}}{\partial x}\right) n^{k}\right\},
$$

where

$$
c^{k}=\left(d^{k} / n^{k}\right)^{1 / 2} \cdot\left(1 / d^{k}\right)^{2} \text {. }
$$

Therefore, $\mathbf{J}_{\mathrm{EF}}^{\mathrm{vUF}, k}$ in (6) is expressed in compact form as

$$
\mathbf{J}_{\mathbf{E F}}^{\mathbf{v U F}, k}=c^{k}\left\{\left(n_{1}^{k} \boldsymbol{\alpha}_{\mathbf{n} 1}+n_{2}^{k} \boldsymbol{\alpha}_{\mathbf{n} 2}\right) d^{k}-\left(d_{1}^{k} \boldsymbol{\alpha}_{\mathbf{d} \mathbf{1}}+d_{2}^{k} \boldsymbol{\alpha}_{\mathbf{d} \mathbf{2}}\right) n^{k}\right\},
$$

where $\boldsymbol{\alpha}_{\mathbf{n} 1}, \boldsymbol{\alpha}_{\mathbf{n} 2}, \boldsymbol{\alpha}_{\mathbf{d} 1}$, and $\boldsymbol{\alpha}_{\mathbf{d} 2}$ are the constant row vectors in (4), respectively. Since $\Delta V U F^{k}$ only depends on the phase voltages at bus $k$, the sensitivity matrix of the VUFs for all 3-ph buses in a power network can be established as shown in (10), which can be simplified to (11). In (10), $\mathbf{O}_{\mathbf{1}_{\mathbf{6}} \mathbf{6}}$ is a zero row vector, and $N_{3 \phi}$ and $N_{T}$ are the number of 3-ph buses and the total number of buses in the power network, respectively: i.e., $N_{T} \geq N_{3 \phi}$. For convenience, Bus 1 is assumed to be a 3-ph bus in (10).

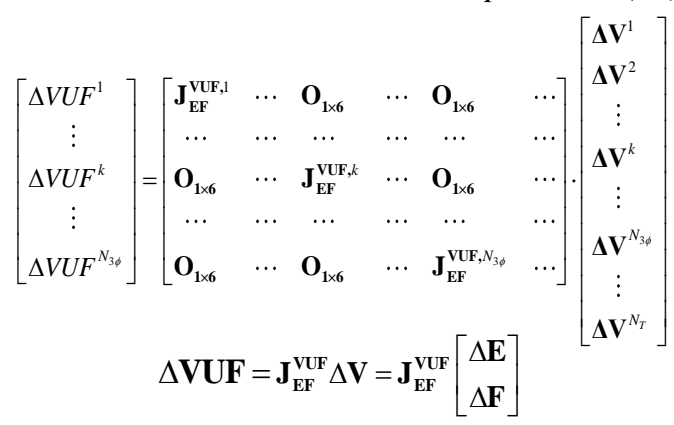

Furthermore, (12) can be derived from (11) by using the sensitivity matrix $\mathbf{J}_{\mathrm{PQ}}^{\mathrm{FF}}$ of complex power injections at all buses with respect to the bus voltages. This enables the estimation of variations in the VUFs with respect to changes in the active and reactive power injections. In (12), $\mathbf{J}_{\mathrm{PQ}}^{\mathrm{EF}}$ can be obtained from general power flow equations with or without consideration of nonlinear equipment, such as voltage regulators or switched capacitors [7], [8]. Note that the sensitivity matrix $\mathbf{J}_{\mathrm{FF}}^{\mathrm{VUF}}$ is independent of nonlinear control actions or equipment operating characteristics, because it is derived from the standard definition of the VUF (i.e., $\left.\left|V_{-}\right| /\left|V_{+}\right|\right)$.

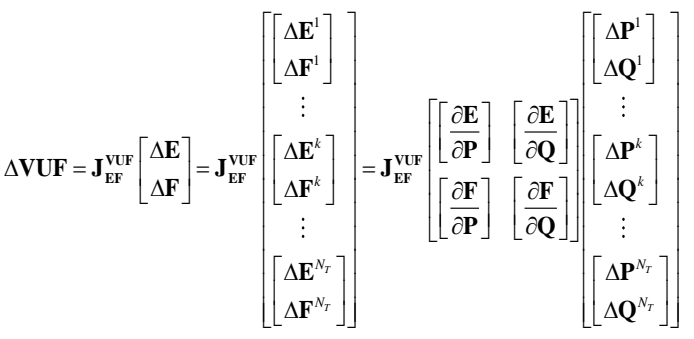

$$
\begin{aligned}
& =\mathbf{J}_{\mathrm{EF}}^{\mathrm{VUF}} \mathbf{J}_{\mathrm{PQ}}^{\mathrm{EF}}\left[\begin{array}{l}
\Delta \mathbf{P} \\
\Delta \mathbf{Q}
\end{array}\right]=\mathbf{J}_{\mathrm{PQ}}^{\mathrm{VUF}}\left[\begin{array}{c}
\Delta \mathbf{P} \\
\Delta \mathbf{Q}
\end{array}\right]
\end{aligned}
$$

The sensitivity matrices $\mathbf{J}_{\mathrm{EF}}^{\mathrm{VUF}}$ or $\mathbf{J}_{\mathrm{PQ}}^{\mathrm{VUF}}$ in (12) can then be effectively used to develop various optimization problems for optimal operations of DERs and controllable loads in distribution networks, taking into account changes in the network power quality (e.g., [9]). The optimization problems can be easily solved with LP, rather than more computationally expensive iterative approaches. Note that in practice, distribution and transmission system operators use LP or MILP algorithms for optimal operation of their power networks. Iterative, brute force approaches such as the BFS algorithm also can be used to estimate $\Delta \mathbf{V U F}$; however, iterative approaches cannot be incorporated into LP or MILP.

\section{CASE Studies ANd Simulation Results}

The IEEE 123 Test Feeder was used as a test bed for the simulation case studies, with the slight modifications discussed in [9] incorporated. Fig. 1 shows that the test bed includes 1-ph and 3-ph buses and, consequently, contains not only 3-ph balanced loads but also 3-ph unbalanced loads and 1-ph loads. The load demand at each bus was scaled so that the total load demand was set to $P_{L}+j Q_{L}=7.04 \mathrm{MW}+j 3.88 \mathrm{MVar}$ (i.e., $p f=$ $0.87)$.

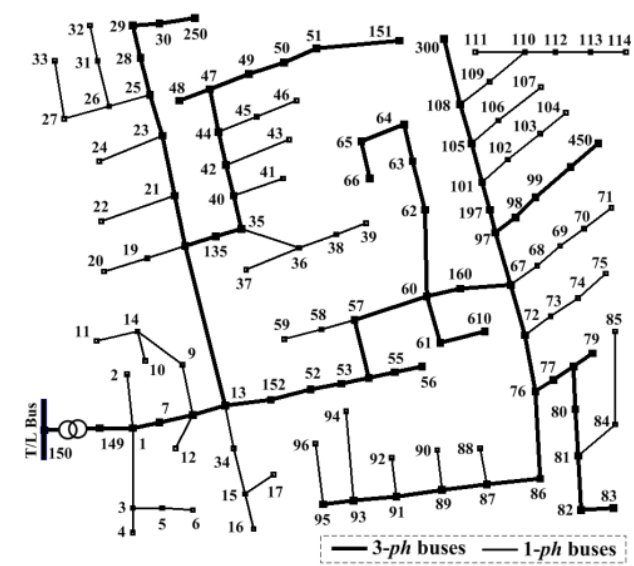

Fig. 1. Test distribution network used in the case studies
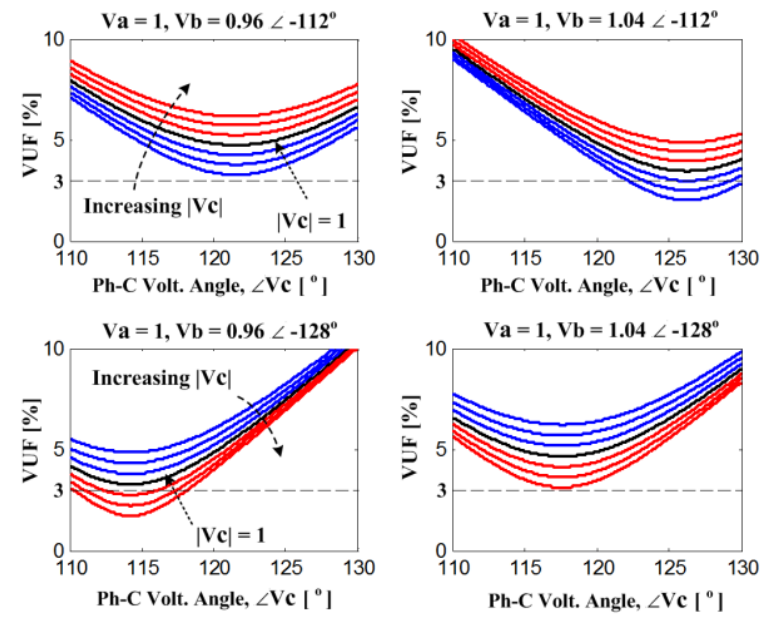

Fig. 2. VUF variations with respect to the voltage magnitudes and angles

Fig. 2 shows example variations in the VUF as the angles and magnitudes of the 3-ph voltages $V_{a}, V_{b}$, and $V_{c}$ are varied. It can be seen that the VUF is a convex function of the voltage magnitudes and angles and, consequently, of the active and reactive power drawn from the network. Fig. 3(a) shows that the maximum and average values of the VUFs at the 3-ph buses increased gradually as the total load demand increased. This is because the unbalanced current flows increased with the 
unbalanced load, leading to larger unbalanced voltage drops. Note that the $x$-axis in Fig. 3 is represented using the ratio $r$ of $\Delta P_{L}$ and $\Delta Q_{L}$ to $P_{L}$ and $Q_{L}$, respectively. In addition, Figs. 3(b) and (c) show $V U F^{\max }$ and $V U F^{\text {avg }}$ for the load variations in phases $\mathrm{B}$ and $\mathrm{C}$, respectively, at 10 arbitrarily selected buses, while keeping the load demands in the other phases fixed. This implies that the VUFs in the test network can be minimized in practice by optimizing the unbalanced power inputs of the 1-ph loads, rather than by simply reducing all their power inputs.
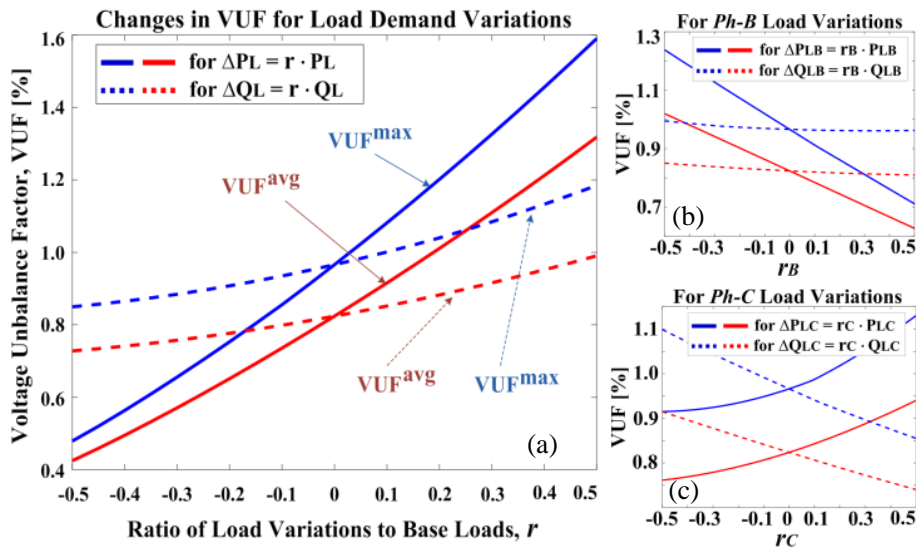

Fig. 3. Variations in the VUF with respect to the changes in active and reactive load demands: (lines) $\Delta P_{L}$ and (dotted lines) $\Delta Q_{L}$

Fig. 4 shows a comparison of the VUFs estimated using (12) and a BFS power flow calculation algorithm [5] (i.e., $\mathbf{V U F}(12)$ and $\mathbf{V U F}$ BFs, respectively) for the case of a $10 \%$ increase in the total load demand. Figs. 4(a) and (b) show the VUFs at all the 3-ph buses for $\Delta P_{L}$ and $\Delta Q_{L}$, respectively. It can be seen that the $\Delta$ VUF estimates calculated using (12) are reasonably accurate.
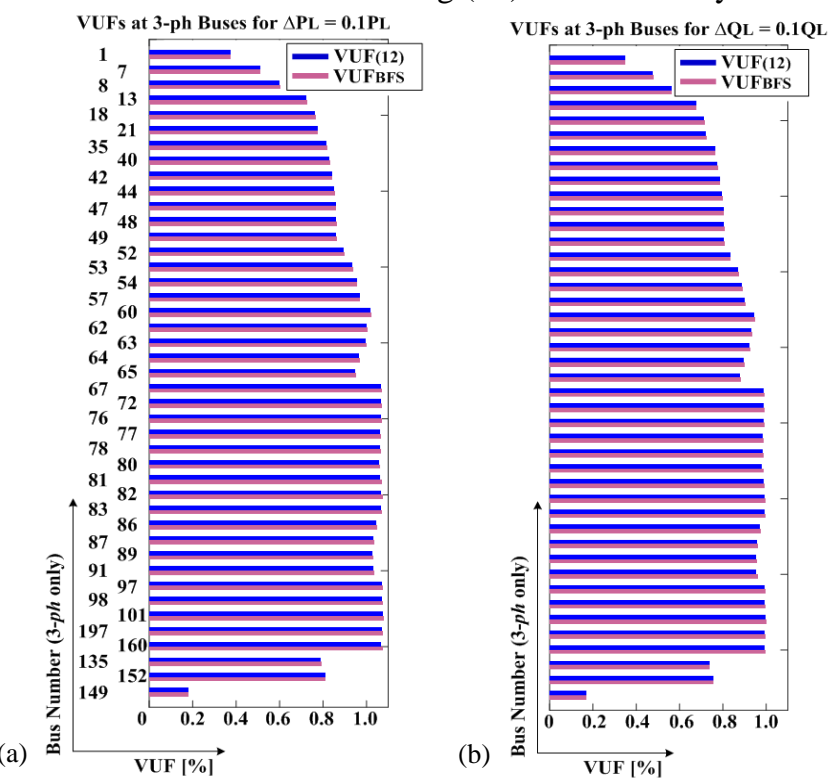

Fig. 4. Comparisons of VUFs at the 3-ph buses obtained using (12) and a BFS power flow algorithm for load variations: (a) $\Delta P_{L}=0.1 P_{L}$ and (b) $\Delta Q_{L}=0.1 Q_{L}$

In addition, Fig. 5(a) shows the maximum and average errors in the VUF estimates (i.e., $\varepsilon^{\max }$ and $\varepsilon^{\text {avg }}$, respectively) at the 3-ph buses for various values of $\Delta P_{L}$ and $\Delta Q_{L}$. The errors were calculated as shown in (13) and (14). In Fig. 5, $\varepsilon^{\max }$ remains less than $2 \%$ when $P_{L}$ and $Q_{L}$ decrease by approximately $33.4 \%$ and $39.8 \%$, respectively, and when $P_{L}$ and $Q_{L}$ increase by approximately $22.5 \%$ and $24.4 \%$, respectively. Moreover, Figs. 5(b) and (c) represent $\varepsilon^{\max }$ and $\varepsilon^{a v g}$ in the VUF estimates for the load variations shown in Figs. 3(b) and (c), respectively. In both cases, $\varepsilon^{\max }$ remains less than $3 \%$ for $30 \%$ load variations, verifying the accuracy of the VUF sensitivity matrix.

As the main aim of this letter is to develop and validate $\mathrm{J}_{\mathrm{FF}}^{\mathrm{VUF}}$ as presented in (12), only constant power loads were considered in the case studies for simplicity. The proposed VUF sensitivity matrix also can be applied to ZIP coefficients models of load devices, resulting in steady-state VUF variations and estimation errors similar to those shown in Figs. 3 and 5, respectively.

$$
\begin{gathered}
\varepsilon^{\max }=\left|\mathbf{V} \mathbf{U} F_{(12)}^{\max }-\mathbf{V} \mathbf{U} \mathbf{F}_{\mathrm{BFS}}^{\max }\right| / \mathbf{V U F}_{\mathrm{BFS}}^{\max }, \\
\varepsilon^{\text {avg }}=\left|\mathbf{V} \mathbf{U} \mathbf{F}_{(12)}^{\text {avg }}-\mathbf{V} \mathbf{U} \mathbf{F}_{\mathrm{BFS}}^{\text {avg }}\right| / \mathbf{V U F}_{\mathrm{BFS}}^{\text {avg }},
\end{gathered}
$$

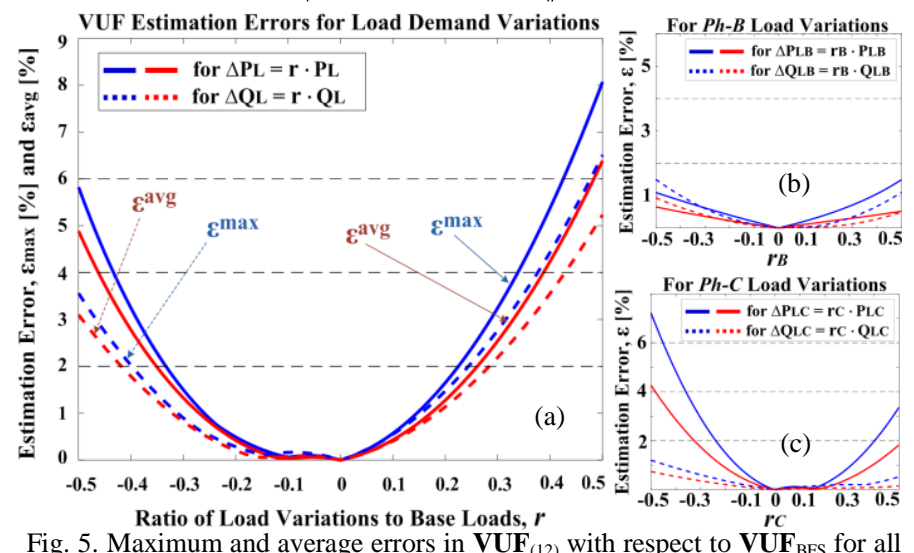
3-ph buses as a function of $\Delta P_{L}$ (lines) and $\Delta Q_{L}$ (dotted lines)

\section{CONCLUSIONS}

In this paper, a sensitivity matrix for VUFs was developed with respect to variations in the unbalanced power inputs of 1-ph and 3-ph loads. Case studies demonstrated the accuracy of the VUF estimation over a wide range of power variations. This shows that the VUF sensitivity matrix can be incorporated into linear optimization problems for optimal operation of DERs and loads in unbalanced distribution networks, considering the improvement in the network power quality. The simulations were run using MATLAB on a four-core, 3.5 GHz CPU, and took insignificant lengths of time to run.

\section{REFERENCE}

[1] A. von Jouanne and B. Banerjee, "Assessment of voltage unbalance," IEEE Trans. Power Del., vol. 16, no. 4, pp. 782-790, Oct. 2001.

[2] M. Savaghebi et al., "Secondary control scheme for voltage unbalance compensation in an islanded droop-controlled microgrid," IEEE Trans. Smart Grid, vol. 3, no. 2, pp. 797-807, Jun. 2012.

[3] A. Siddique et al., "Effects of voltage unbalance on induction motors," in Conf. Rec. IEEE Int. Symp. Elect. Insul., 2004, pp. 26-29.

[4] J. K. Kang and S. K. Sul, "Control of unbalanced voltage PWM converter using instantaneous ripple power feedback," in Proc. IEEE PESC, 1997, pp. 503-508.

[5] G. W. Chang, S. Y. Chu, and H. L. Wang, "An improved backward/forward sweep load flow algorithm for radial distribution systems," IEEE Trans. Power Syst., vol. 22, no. 2, pp. 882-884, May 2007.

[6] P. Pillay and M. Manyage, "Definitions of voltage unbalance," IEEE Power Eng. Rev., vol. 21, no. 5, pp. 49-51, May 2001.

[7] H. Ahmadi, J. R. Marti, and A. von Meier, "A linear power flow formulation for three-phase distribution systems," IEEE Trans. Power Syst., vol. 31, no. 6, pp. 5012-5021, Nov. 2016

[8] B. Stott, "Review of load-flow calculation methods," Proc. IEEE, vol. 62, no. 7, pp. 916-929, Jul. 1974.

[9] Y. Kim et al., "Optimal use of thermal energy storage resources in commercial buildings through price-based demand response considering distribution network operation," Appl. Energy, vol. 193, pp. 308-324, 2017. 